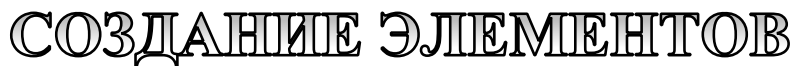

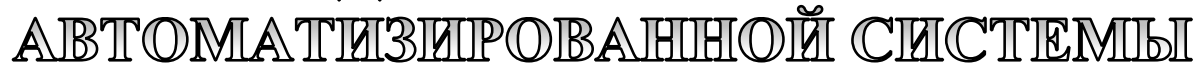

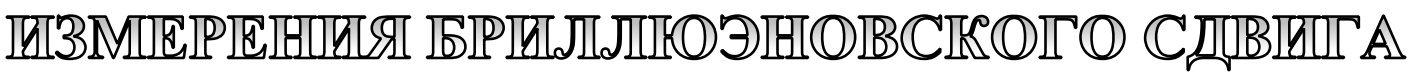

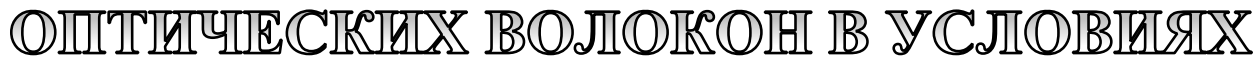

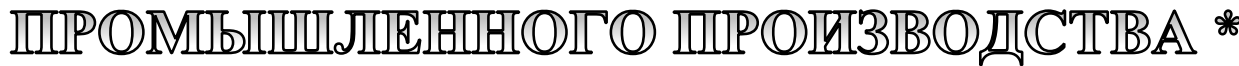

А.И. Кривошеев, Пермский федеральный исследовательский иентр УрО РАН

Ю.А. Константинов, Пермский федеральный исследовательский иеетр УрО РАН

\section{Для цитирования:}

Кривошеев А.И., Константинов Ю.А. Создание элементов автоматизированной системы измерения бриллюэновского сдвига оптических волокон в условиях промышленного производства // Вестник Пермского федерального исследовательского центра. - 2021. - № 4. - С. 37-48. https://doi.org/10.7242/2658-705X/2021.4.4

Представлено современное рассмотрение проблемы создания автоматизированной системы контроля качества специальных оптических волокон, сохраняющих состояние поляризации излучения. Представлена имитационная модель рефлектометрии, учитывающая продольное скручивание оптического волокна. Изложен принцип работы системы с учетом использования нового метода обработки спектров рассеяния Мандельштама-Бриллюэна в совокупности с уже известными методами, объединенными в нейронную сеть, для увеличения точности системы.

Ключевые слова: автоматизированная система измерений (АСИ), оптическая рефлектометрия, оптические волокна, автоматизированный контроль, бриллюэновская рефлектометрия, поляризаџия.

\section{Введение}

Современные тенденции развития волоконно-оптических технологий и датчиков на их основе требуют все более строгого внимания к контролю эксплуатационных параметров оптических волокон на всех стадиях производства. Разработка автоматизированных систем анализа параметров оптических волокон, поэтому, является важной задачей, которой уже по- священо немало работ [1-3]. Оптическая рефлектометрия временной области (OTDR - optical time domain reflectometry), наряду с рефлектометрией частотной области (OFDR - optical frequency domain reflectometry), широко применяется в промышленном производстве оптических волокон в качестве систем распределенного мониторинга параметров оптического волокна [4-6]. В общих чертах, принцип

* Работа выполнена в рамках государственного задания (регистрационный номер № AАAА-А19119042590085-2. 
работы таких систем распределенного мониторинга основывается на регистрации различных компонент обратнорассеянного излучения в каждой точке оптического волокна и изучении его спектральных, фазовых, поляризационных и иных свойств, а также математической обработке сигнала.

Одним из особенно актуальных вопросов становится автоматизация контроля производства специальных оптических волокон, например, сохраняющих поляризацию оптического излучения. Контроль параметров таких волокон является непростой задачей для классических систем распределенного анализа в связи с имеющейся в волокне анизотропией оптических свойств, влияющих на распространение излучения.

Особое внимание современных исследователей уделено системам, основанным на вынужденном и спонтанном рассеянии Мандельштама-Бриллюэна. Такие системы работают по принципу рассеяния света на акустическом фононе, присутствие которого имеет место в оптическом волокне, по которому распространяется зондирующее излучение. Температурное и деформационное воздействие на оптическое волокно приводит к изменению скорости звука в стекле, из которого оно изготовлено, что, в свою очередь, ведет к смещению частотной компоненты обратно-рассеянной волны. Именно координата максимума обратноотраженной спектральной компоненты сигнала и является носителем информации о величине воздействия на оптическое волокно. Эту частотную координату принято называть сдвигом бриллюэновской частоты (BFS - Brillouin Frequency Shift).

Методом бриллюэновской рефлектометрии потенциально возможно регистрировать очень малые величины температурных и деформационных воздействий. Однако после первичного аналого-цифрового преобразования спектры, регистрируемые системой, представляют собой дискретные функции, содержащие цифровой шум, в которых искомая спектральная компонента может быть скрыта.
Немало внимания также уделено современным подходам к увеличению точности и снижению шумов при получении и обработке бриллюэновских рефлектограмм и спектров.

Современным стандартом в системах мониторинга на основе бриллюэновской рефлектометрии стала аппроксимация лореневой функцией (Lorentzian Curve Fitting - LCF) [7, 8] - метод, основанный на итерационном подборе аналитической функции Лоренца и её сравнении с полученным сигналом. Конкуренцию ему составляют корреляционные методы, недавно представленные в литературе. Они менее сложны в реализации, и скорость их работы выше. Исходя из точностных параметров, ситуация не так однозначна. На различных реализациях спектров излучения, имеющих различные соотношения сигнал/шум, а также возможные искажения, разные методы обработки могут давать разную точность определения максимума.

Задача настоящей работы состоит в проработке основных элементов для создания автоматизированной системы контроля качества специальных оптических волокон, сохраняющих состояние поляризации излучения, при помощи современных подходов обработки информации, полученной с систем оптической рефлектометрии.

\section{Источники спектральных шумов и искажений}

В процессе эксплуатации оптических волокон, сохраняющих состояние поляризации, нередко возникают ситуации, когда происходит необъяснимый скачок/падение поляризационной экстинкции, которая является основной характеристикой способности оптического волокна сохранять состояние поляризации вводимого излучения.

Для того чтобы попытаться объяснить подобные явления, был выбран один из трудно регистрируемых дефектов, а именно - продольная скрутка оптического волокна. Она может образоваться при укладке анизотропного волоконного сен- 
сора в исследуемый объект и оказать еще более существенное влияние на показания устройства после полимеризации пропиточного материала.

Для описания поляризационных неоднородностей был выбран матричный формализм Джонса [9]. Для этого необходимо представить волоконный световод как последовательность повернутых относительно друг друга полупрозрачных фазовых пластинок, каждая из которых будет обладать собственной анизотропией.

Матрица Джонса фазовой пластинки длиной $L$ с показателями преломления $n_{1}$ и $n_{2}$ (m-го участка волокна), как известно, в системе координат с осями, параллельными осям пластинки, имеет вид

$$
M_{m}=\left[\begin{array}{cc}
1 & 0 \\
0 & e^{-i \Delta \varphi}
\end{array}\right],
$$

где $\Delta \varphi=\frac{2 \pi L(n 2-n 1)}{\lambda}-$ фазовая задержка медленной компоненты волны, обусловленная разностью показателей преломления.

Поскольку рассматриваемый участок волокна ввиду закрутки повернут на определенный угол $\boldsymbol{\theta}_{m}$ (пропорциональный расстоянию от конца волокна до участка, $\boldsymbol{\theta}_{m}=\alpha L_{m}$, где $\alpha-$ коэффициент закрутки, в рад/км, $L_{m}-$-пространственная координата $m$-го участка), в нормальной системе координат матрица Джонса преобразуется соответствующим образом:

$T_{m}=T_{\text {rot }}\left(\boldsymbol{\theta}_{m}\right) M_{m} T_{\text {rot }}\left(-\boldsymbol{\theta}_{m}\right)$,
где $T_{\operatorname{rot}(\theta)}=\left[\begin{array}{cc}\cos (\theta) & \sin (\theta) \\ -\sin (\theta) & \cos (\theta)\end{array}\right]-$ матрица поворота.

Следует также учесть, что при обратном распространении световой волны волокно становится закрученным в другую сторону, так что матрица Джонса того же участка волокна будет иметь несколько другой вид:

$$
\mathrm{T}_{\mathrm{m}}{ }^{*}=\mathrm{T}_{\text {rot }}\left(\boldsymbol{\theta}_{\mathrm{m}}\right) \mathrm{M}_{\mathrm{m}} \mathrm{T}_{\text {rot }}\left(\boldsymbol{\theta}_{\mathrm{m}}\right) .
$$

Пространственную (временну́ю) развертку мощности обратного рассеяния в момент времени $t_{n}$ в подобной системе можно представить как

$$
E_{n-1}=F_{0} b_{n-1} \Pi_{i=1}^{n}\left(f_{i}\right)^{2},
$$

где $E_{n-1}$ - уровень обратно-рассеянного излучения на фрагменте $(n-1) ; F_{0}-$ уровень излучения, введенного в световод; $b$ и $f$ - коэффициенты, определяющие параметры отражения и затухания на заданном фрагменте. Предположим, что схожую идеологию рассмотрения нестационарного процесса можно перенести на поляризационные рефлектограммы. Тогда выражения для обратнорассеянного линейно-поляризованного света (ввод излучения под 0 градусов) будет выглядеть следующим образом:

$$
\left[\begin{array}{l}
E_{x(0)}(z) \\
E_{y(0)}(z)
\end{array}\right]=\left[\begin{array}{cc}
E x t_{p o l} & 0 \\
0 & 0
\end{array}\right] \Pi_{m=1}^{Z} T_{m=z}^{*} \Pi_{m=z}^{1} T_{m}\left[\begin{array}{l}
1 \\
0
\end{array}\right],(5)
$$

где $E x t_{p o l}$ - поляризационная экстинкция поляризатора, присутствующего в схеме рефлектометра. Полученные $E_{x(0)}(\mathrm{z})$ и $E_{y(0)}(\mathrm{z})$ уместно использовать для вычисления сигнала обратного рассеяния для случая частотной области.

На рис. 1 представлены две результирующие рефлектограммы (векторные суммы) для двух случаев: угол закрутки равен 0 рад/км и 1 рад/км.

В результате моделирования метода частотной рефлектометрии для случая оптического волокна, сохраняющего поляризацию излучения, было показано, что даже незначительные закрутки (порядка 1 рад/км) могут приводить к существенным искажениям на рефлектограммах оптических волокон.

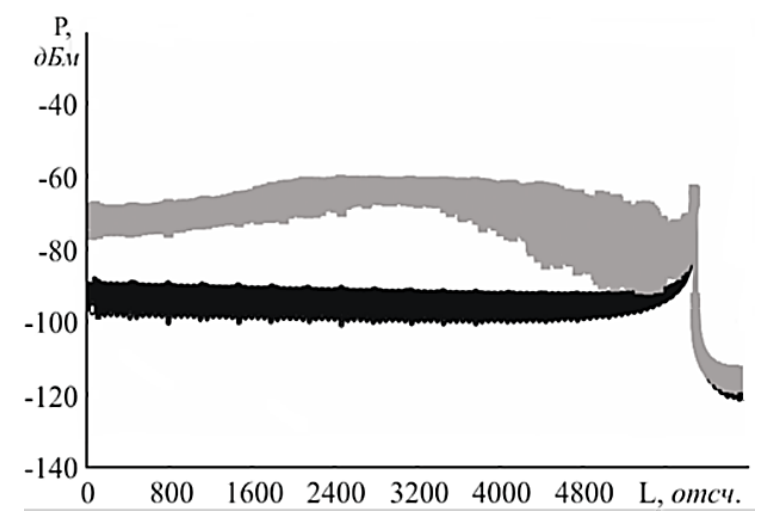

Рис. 1. Смоделированные рефлектограммы частотной области для волокна с осевым кручением (верхняя) и без него (нижняя) 
Стоит отметить, что, хотя моделирование и проводилось для случая частотной области, все эффекты, описанные выше, имеют место и для случая рефлектометрии временной области. Это позволяет предположить, что локальные изменения поляризационной экстинкции могут оказывать воздействие на параметры спектральных компонент излучения, что, в свою очередь, приведет к неверной обработке полученного сигнала системами мониторинга.

Данная модель позволит оценивать возможность исследования образца оптического волокна с помощью представленной автоматизированной системы.

\section{Калибровка системы измерения}

Одно из направлений волоконного мониторинга, активно исследуемое в настоящее время многими авторами, - одновременное и независимое детектирование температуры и деформации в оптическом волокне. Учеными опубликовано множество работ с применением различных способов получения информации о температуре и деформации в оптическом волокне; к таким способам относятся внесение изменений в конструкцию волоконного тракта, аппаратную часть прибора, а также постобработка полученных рефлектограмм.

Тем не менее, само по себе получение спектральных компонент не гарантирует получение достоверных сведений о состоянии оптического волокна. Для этого необходима предварительная калибровка системы с учетом известного воздействия на определенное оптическое волокно.

Для оценки влияния точности калибровки на погрешность сенсора была выбрана задача одновременного детектирования температуры и деформации в волокне, сохраняющем состояние поляризации излучения, с использованием бриллюэновского анализатора (BOTDA Brillouin Optical Time Domain Analyizer).

В общем случае, изменение сдвига бриллюэновской частоты в присутствии температурных или деформационных воздействий можно описать формулой

$$
\left(\begin{array}{c}
\Delta f_{x} \\
\Delta f_{y}
\end{array}\right)=A\left(\begin{array}{c}
\Delta T \\
\Delta \varepsilon
\end{array}\right),
$$

где $\Delta f_{x}, \Delta f_{y}$ - изменения частоты бриллюэновского сдвига для двух осей, обусловленные температурными $\Delta T$ и механическими $\Delta \varepsilon$ воздействиями, то есть разница между бриллюэновским сдвигом при воздействии $\Delta T, \Delta \varepsilon$ и при отсутствии воздействий; А - матрица коэффициентов чувствительности: $A=\left\|\begin{array}{ll}a_{11} & a_{12} \\ a_{21} & a_{22}\end{array}\right\|$.

Коэффициенты чувствительности матрицы находятся путем проведения калибровочного эксперимента с оптическим волокном. Калибровочное измерение состояло из двух этапов. Первый этап представляет собой получение температурных зависимостей бриллюэновского сдвига для двух состояний поляризации, а второй - деформационных зависимостей (растяжение волокон).

Для опыта с термическими испытаниями зависимость бриллюэновских сдвигов $f_{x}$ и $f_{y}$ от температуры показана на рис. 2. Зависимости для опыта с натяжением волокон выглядят аналогично, имея лишь несколько иной наклон по отношению к оси абсцисс.

По тангенсам угла наклона кривых были получены значения коэффициентов матрицы:

$$
A=\left(\begin{array}{ll}
1,271 M H_{Z} / K & 0,043 M H_{Z} / \mu \varepsilon \\
1,159 M H_{Z} / K & 0,044 M H_{Z} / \mu \varepsilon
\end{array}\right) .
$$

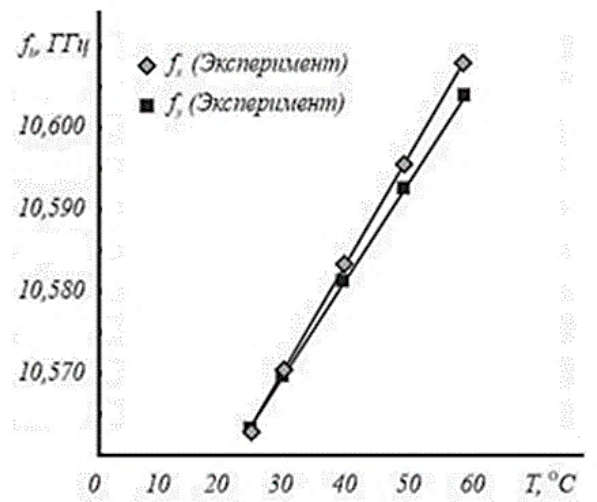

Рис. 2. Зависимость бриллюэновских сдвигов $\Delta f_{x} u \Delta f_{y}$ от температуры, полученная во время калибровочного эксперимента 
По заданным значениям $\Delta \mathrm{T}$ и $\Delta \varepsilon$ с использованием данных коэффициентов рассчитывались бриллюэновские сдвиги $\Delta f_{x}$ и $\Delta f_{y}$. При обратном пересчете $\Delta f_{x}$ и $\Delta f_{y}$ с использованием тех же коэффициентов матрицы, естественно, получаются исходные $\Delta \mathrm{T}$ и $\Delta \varepsilon$. Однако при внесении изменений в коэффициенты матрицы будут получены некие другие значения $\Delta \mathrm{T}^{\prime}$ и $\Delta \varepsilon^{\prime}$. Зависимость средней по модулю разницы между $\Delta \mathrm{T}$ и $\Delta \mathrm{T}^{\prime}$ от вариации коэффициентов матрицы представлена на рис. 3. Видно, что результаты неодинаково чувствительны к разным коэффициентам матрицы, но общий вид зависимости одинаков - чем больше погрешность коэффициентов матрицы, тем менее точно определяется температура. Для достижения точности измерений температуры в несколько процентов погрешности коэффициентов не должны превышать долей процента.

Зависимость погрешности определения деформации, не представленная на рис. 3, носит аналогичный характер.

Далее необходимо определить основные параметры, влияющие на точность получаемых калибровочных коэффициентов. Основными параметрами в данном случае выбраны соотношение сигнал/шум полученных спектров бриллюэновского рассеяния, а также шаг сканирования спектра по частоте.

На рис. 4 представлены смоделированные зависимости погрешности определения температуры оптического волок-

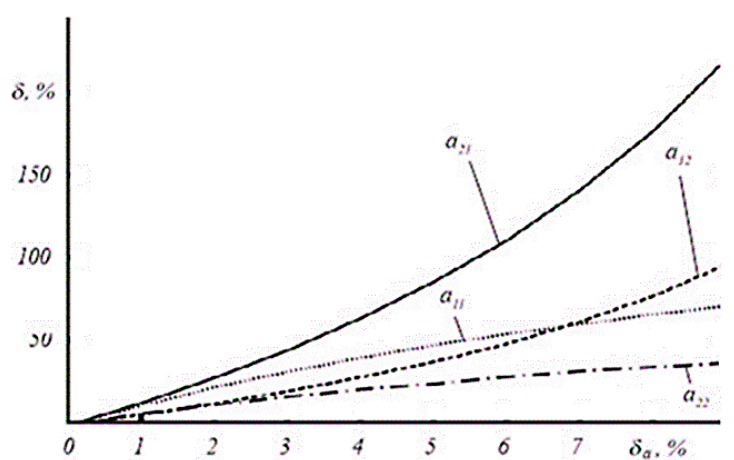

Рис. 3. Зависимость погрешности расчетного эксперимента от погрешности ввода калибровочных коэффициентов на для различных соотношений сигнал/шум от величины шага сканирования по спектру.

По полученным графикам становится видно, что даже небольшое ухудшение соотношения сигнал/шум приводит к значительным погрешностям определения величин воздействия окружающей среды.

\section{Определение BFS в АСИ}

Как было замечено ранее, корреляционные методы могут быть использованы в качестве альтернативы LCF методу. Ранее было показано [10], что метод «обратной» корреляции (BWC - backward correlation), детально описанный в $[11,12]$, показал хорошую эффективность в нахождении максимума бриллюэновского спектра в условиях низкого соотношения сигнал/шум, искажений формы спектра, а также дефектов оцифровки.

Предположим, что мы имеем спектр, полученный в результате измерения, в виде дискретного набора выборок, состоящий из $2 N+1$ пар $\left[f_{0}+i * \Delta f, P_{i}\right]$, где $i$ - номер выборки в спектре, изменяющийся от 0 до $2 N, f_{0}$ - минимальная частота спектра, $\Delta f$ - шаг сканирования по частоте, определяемый аппаратной дискретизацией, $P_{i}$ - зарегистрированная плотность мощности обратного рассеяния на частоте $f_{i}=f_{0}+i * \Delta f . P_{i}$ состоит из двух частей - собственно полезного сигнала и шума, $P_{i}=P_{i}^{s}+P_{i}^{n}$. При отсутствии шумовой составляющей, например при $P^{n}{ }_{i}=0$, нахождение максимальной плотно-

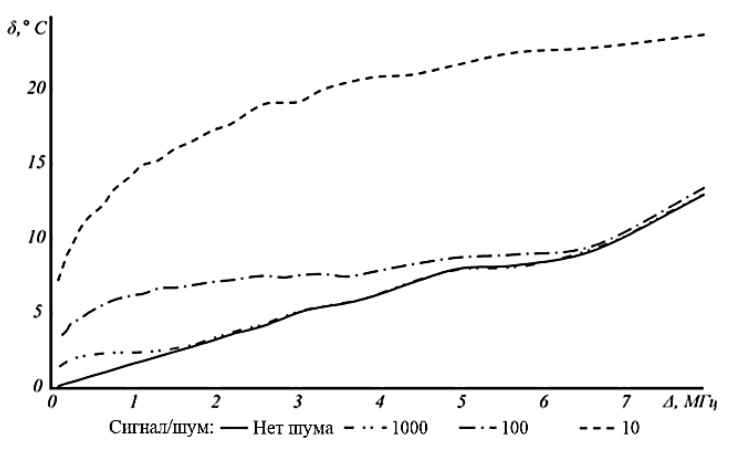

Рис. 4. Полученные моделированием погрешности определения температуры в зависимости от шага сканирования и соотношения сигнал-шум 
сти мощности дало бы центральную частоту BGS $f_{b}$ (BFS) с точностью до ошибки выборки, которая в среднем составляет $\Delta f / 4$. Однако в работе [13] было показано, что наличие даже небольшого шума (SNR $<20$ дБ) приводит к тому, что погрешность в основном определяется шумом в спектре и слабо уменьшается с уменьшением $\Delta f$.

Пусть «обратный» сигнал $P^{\prime}$ описан как $P_{i}^{\prime}=P_{2 N-i}$, а «обратный и смещенный» сигнал $P^{\prime \prime}(k)$ - как $P^{\prime \prime}{ }_{i}=P^{\prime}{ }_{i-k}$, учитывая, что $P^{\prime \prime}=0$, если $[i-k]$ находится вне диапазона $[0,2 N]$. Здесь $k$ - сдвиг сигнала, который может принимать все виды целочисленных значений от $-2 \mathrm{~N}$ до $2 \mathrm{~N}$.

Свертка сигналов $P$ и $P^{\prime \prime}$ может быть описана следующим образом:

$$
\begin{aligned}
& X=\sum_{i=0}^{2 N} P_{i} * P^{\prime \prime}{ }_{i}=\sum_{i=0}^{2 N} P_{i}^{S} * P_{i}^{\prime \prime}+ \\
& +\sum_{i=0}^{2 N} P_{i}^{n} * P_{i}^{\prime \prime}{ }_{i}+\sum_{i=0}^{2 N} P_{i}^{S} * P_{i}^{\prime \prime}{ }_{i}+\sum_{i=0}^{2 N} P_{i}^{n} * P_{i}^{\prime \prime}{ }_{i} .
\end{aligned}
$$

Второе и третье слагаемые должны быть равны нулю с точностью до статистической погрешности, так как шумовая и полезная составляющие сигнала независимы. Четвертый член также должен быть близок к нулю, поскольку значения шума умножаются в разных точках. Что касается первого члена, то чем ближе максимумы $P^{\mathrm{s}}$ и $P^{\prime \prime s}$ зашумленного спектра друг к другу, тем он больше. Построив график зависимости $X$ от величины сдвига $k$ (рис. 5, нижняя кривая) и определив, при каком сдвиге $k_{0}$ значение $X$ достигает своего максимума (при его наличии), можно получить частоту, соответствующую максимуму BGS $P^{\mathrm{s}}$ : $f_{b}=f_{0}+\left(N-k_{0} / 2\right) * \Delta f, \quad$ с точностью до ошибки дискретизации.

В $[10,11]$ максимум функции «обратной» корреляции (см. рис. 5, нижняя кривая) определялся путем прямого перебора - не самый лучший метод, как уже показано ранее. Для того чтобы применять другие техники поиска максимума, необходимо определить форму получаемой в результате корреляции функции. Свертка двух функций Лоренца, сдвинутых относительно друг друга на величину $\delta$, задается (здесь мы пренебрегаем эффектами дискретизации и переходим от суммы к интегрированию):

$I(f)=\int_{-\infty}^{\infty} \frac{w^{2} \pi^{-2}}{\left[\left(f-f_{b}\right)^{2}+w^{2}\right]\left[\left(f-f_{b}-\delta\right)^{2}+w^{2}\right]} d f,(9)$ где $w$ - ширина спектра, $f_{b}-$ BFS. Для простоты, на данном этапе, зададим $f_{\mathrm{b}}$ равным нулю. Отсюда

$$
\begin{aligned}
& I(f)=\frac{w^{2}}{\pi^{2}} \int_{-\infty}^{\infty} \frac{d f}{\left[f^{2}+w^{2}\right]\left[(f-\delta)^{2}+w^{2}\right]}= \\
& =\frac{w^{2}}{\pi^{2}} \int_{-\infty}^{\infty} \frac{d f}{\left[\left(f-z_{1}\right)\left(f-z_{2}\right)\right]\left[\left(f-z_{3}\right)\left(f-z_{4}\right)\right]} .(10)
\end{aligned}
$$

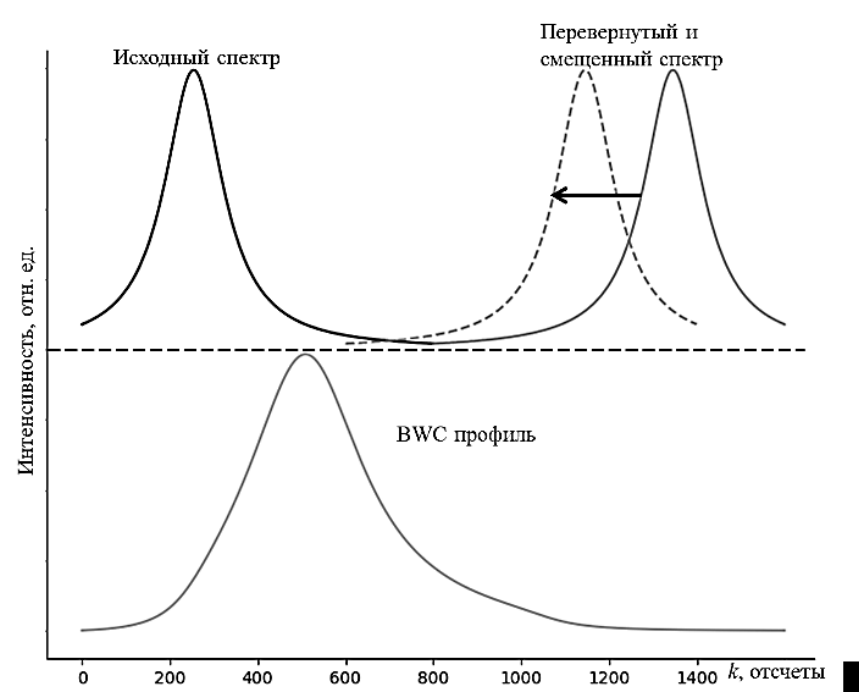

Рис. 5. Принщипиальная схема, описывающчая принщฺи работы нового метода 
Здесь $z_{1-2}= \pm i^{*} w, z_{3-4}=\delta \pm i^{*} w-$ особенные точки, в которых знаменатель обращается в ноль.

Возьмем замкнутую кривую сечением $[-R, R]$, а полуокружность находится в полуплоскости, где мнимая часть положительна, как показано на рис. 6. Согласно теореме о вычетах, интеграл рассматриваемой функции по замкнутой кривой равен $2 \pi i \sum \operatorname{res} f$ и суммирование выполняется по всем особенным точкам внутри кривой, т.е. по $\mathrm{z}_{1}$ и $\mathrm{z}_{3}$. В пределе $R \rightarrow \infty$ интеграл по полукругу стремится к нулю (поскольку длина полукруга пропорциональна только $\mathrm{R}$, а подынтегральное значение равно $1 / R^{2}$ ). Очевидно, что интеграл по сечению $[-R, R]$ стремится к требуемому значению $I$. Тогда вычеты в точках $z=z_{1}$ и $z=z_{3}$

$$
\begin{aligned}
& \operatorname{Res} f \mid z=i w=\frac{1}{(i w+i w)\left[(i w-\delta)^{2}+w^{2}\right]}= \\
& =\frac{1}{2 i w\left[\delta^{2}-2 i w \delta\right]},
\end{aligned}
$$

$$
\begin{aligned}
& \operatorname{Res} f \mid z=\delta+i w= \\
& =\frac{1}{(\delta+i w-\delta+i w)\left[(i w-\delta)^{2}+w^{2}\right]}= \\
& =\frac{1}{2 i w\left[\delta^{2}-2 i w \delta\right]},
\end{aligned}
$$

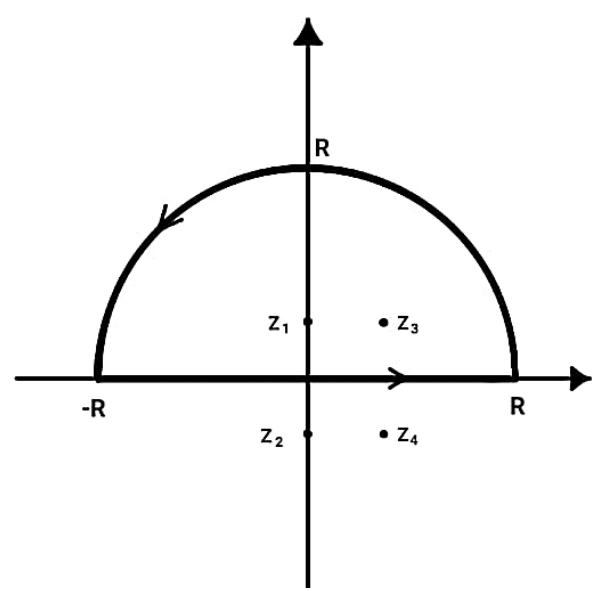

Рис. 6. Иллюстрациия процеесса интегрирования
Таким образом, можно записать:

$$
\begin{aligned}
& I=2 i \frac{w^{2}}{\pi}\left[\frac{1}{2 i w\left[-2 i w \delta+\delta^{2}\right]}+\frac{1}{2 i w\left[2 i w \delta+\delta^{2}\right]}\right]= \\
& =\frac{w}{\pi}\left[\frac{2 \delta^{2}}{\left[2 i w \delta-\delta^{2}\right]\left[2 i w \delta+\delta^{2}\right]}\right]= \\
& =\frac{(2 w)}{\pi}\left[\frac{1}{\left[(2 w)^{2}+\delta^{2}\right]}\right],
\end{aligned}
$$

$I(\delta)=\frac{(2 w)}{\pi}\left[\frac{1}{\left[(2 w)^{2}+\delta^{2}\right]}\right]=\frac{W}{\pi}\left[\frac{1}{\left[W^{2}+\delta^{2}\right]}\right]$,

где $W$ - ширина профиля BWC.

Очевидно, что выражение (14) также является функцией Лоренца и, следовательно, имеет четко локализованный максимум, связанный с искомым значением.

Для оценки точности метода «обратной» корреляции сгенерирован набор спектров, содержащих дефекты двух видов. Первые имитируют шум регистраторов и приводят к случайной вариации от спектра к спектру отношения сигнал/шум в диапазоне от 2 до 20 дБ. Вторые представляют собой сбои оцифровки, приводящие к появлению случайных по локации и продолжительности провалов дискретной функции до нулевого значения. Такая дискретная функция может быть выражена следующим образом:

$$
\begin{aligned}
& P(f)= \\
& =\left(P_{N}+\frac{W}{\pi}\left[\frac{1}{\left[W^{2}+f^{2}\right]}\right]\right)\left[1+\frac{(f-\mu)|f-v|}{(f-v)|f-\mu|}\right],
\end{aligned}
$$

где $\mu$ - частотная координата начала цифрового провала сигнала; $v$ - частотная координата конца цифрового провала сигнала, причем $v$ всегда больше $\mu$ и задана случайно в пределах полезного сигнала спектра; $W$ - масштабный коэффициент спектральной функции, отвечающий за ширину спектра и амплитуду полезного сигнала; $P_{N}-$ амплитуда шумовой составляющей сигнала. 
Далее осуществлялась математическая обработка спектров тремя методами: аппроксимацией лоренцевой функцией (LCF), классическим взаимно-корреляционным методом (CCM), а также разработанным методом «обратной» корреляции (BWC).

Дефект, описанный выше и представленный как локальное обращение исследуемой функции в ноль, может быть обусловлен не только дефектами оцифровки, но и некорректной работой алгоритма сканирования по спектру. Его протяженность и локация в спектре могут быть совершенно различными.

Далее рассмотрено два случая: первый описывает появление такого дефекта в спектральных областях, не содержащих искомый максимум; второй же, напротив, предполагает, что максимальная частота спектра всегда находится в границах провала. Для каждого описанного случая возникновения артефактов в спектре было сгенерировано по одной тысяче спектров.

В табл. 1 приведена информация об эффективности всех трех методов при разных отношениях сигнал/шум. В каждом случае спектры были поделены на три группы по соотношению сигнал/шум (SNR). В первую группу вошли спектры с низким SNR (до 5 дБ), во вторую - со средним SNR (5-10 дБ), а в третью - с высоким SNR (> 10 дБ).

Таблицุа 1

Эфффективность нахождения максимума бриллюэновского спектра

\begin{tabular}{|c|c|c|c|c|}
\hline \multirow{2}{*}{ Метод } & \multirow{2}{*}{$\begin{array}{c}\text { SNR, } \\
\mathrm{dB}\end{array}$} & \multicolumn{3}{|c|}{ BFS стандартное отклонение, } \\
\cline { 3 - 5 } & & Без & Случай & Случай \\
& & артесракта & 1 & 2 \\
\hline \multirow{3}{*}{ LCF } & $<5$ & 1,12 & 10,68 & 7893,28 \\
& $5-10$ & 0,24 & 7,55 & 4076,74 \\
& $>10$ & 0,04 & 6,19 & 316,42 \\
\hline \multirow{3}{*}{ BWC } & $<5$ & 2,17 & 9,12 & 9,69 \\
& $5-10$ & 0,74 & 6,18 & 7,53 \\
& $>10$ & 0,14 & 5,83 & 7,68 \\
\hline \multirow{3}{*}{ CCM } & $<5$ & 0,46 & 7,61 & 20,39 \\
& $5-10$ & 0,19 & 6,54 & 19,51 \\
& $>10$ & 0,09 & 6,37 & 16,13 \\
\hline
\end{tabular}

Как уже отмечалось выше, традиционный метод аппроксимации лоренцевой функцией относительно эффективен при высоких отношениях сигнал/шум в случае, если провал не попадает на искомую центральную область спектра. В остальных случаях более эффективны корреляционные методы. Тогда же, когда провал находится в области пика, самым эффективным становится метод обратной корреляции; классический корреляционный метод демонстрирует чуть меньшую точность; в то время как метод аппроксимации лоренцевой функцией полностью теряет свою актуальность в столь экстремальных условиях.

Стоит отметить, что в реальных системах мониторинга, основанных на использовании рассеяния МандельштамаБриллюэна, артефакты, о которых говорилось выше, встречаются достаточно редко в коммерческом оборудовании, но могут обнаруживаться при экспериментах на макетных установках. Несомненно, стоит рассмотреть случаи, когда артефакт проявляется в спектре с некоторой долей вероятности. Прогнозируемое стандартное отклонение $E$ измерения BFS в условиях возникновения артефакта с вероятностью Р можно оценить приближенно следующим образом:

$$
E=E_{0}(1-P / 100)+E_{a}(P / 100),(16)
$$

где $E_{0}-$ стандартное отклонение в условиях отсутствия артефактов; $E a$ - стандартное отклонение в условиях наличия артефакта.

В результате расчета точности системы были получены графики, представленные на рис. 7. Согласно этим графикам, начиная с 9\%-ной вероятности возникновения артефакта (выделенная кружком область), метод BWC показывает лучшие результаты по точности определения максимума бриллюэновского спектра в сравнении с методом CСM. Также для качественной оценки эффективности определения максимума бриллюэновского спектра на рис. 7 представлена аналогичная зависимость для метода LCF. 


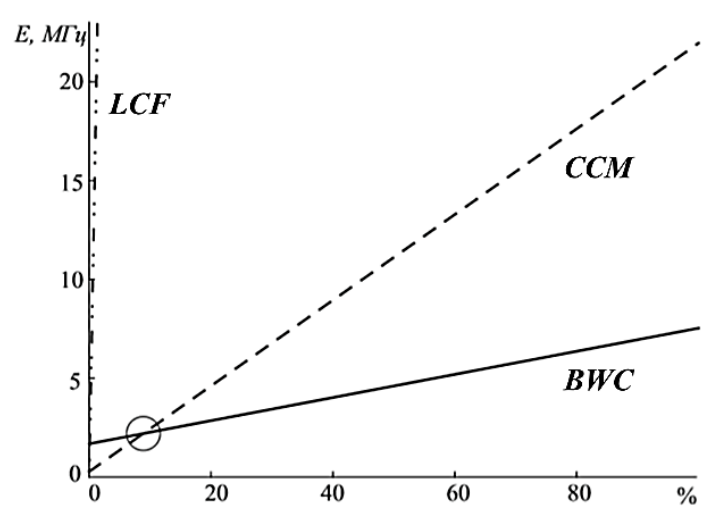

Рис. 7. Стандартное отклонение определения максимума $B G S$ в зависимости от вероятности появления артефакта

Также были проведены исследования реальных спектров бриллюэновского рассеяния при сравнительно высоких SNR. Результаты представлены на рис. 8 .

На основании вышеуказанного можно сделать вывод, что разработанный метод «обратной» корреляции действительно способен обеспечивать лучшую точность определения максимума бриллюэновского спектра в условиях высокой зашумленности и наличия дефектов в спектрах.

Метод BWC может быть потенциально применен в таких приложениях, как разделение температур и деформаций в волокнах, сохраняющих поляризацию излучения [13], где требуется с высокой точностью разделить бриллюэновские спектры двух поляризационных осей оп- тического волокна; распределенное измерение двулучепреломления в волокнах, сохраняющих состояние поляризации [11]; кроме того, потенциальные преимущества данный метод может предоставить при рефлектометрическом исследовании нестандартных оптических волокон с высокими потерями, к каким также относится активное волокно [14].

\section{Концепция автоматизированной системы контроля}

Использование нескольких методов для определения максимума бриллюэновского спектра дает основания полагать, что каждый из них, пусть и с некоторой ошибкой, несет в себе полезную информацию об истинном положении максимума. Простое вычисление среднего арифметического в этой ситуации не принесет должного результата, поскольку полагается, что каждый метод имеет равный вес, в то время как в зависимости от выходного результата, а также других параметров, весовое значение того или иного метода может меняться.

Предполагаемым решением в такой ситуации может стать нейронная сеть, которая в процессе обучения способна настроить весовые связи таким образом, чтобы наилучшим способом выявить вклад каждого метода в определение истинного положения максимума спектра.

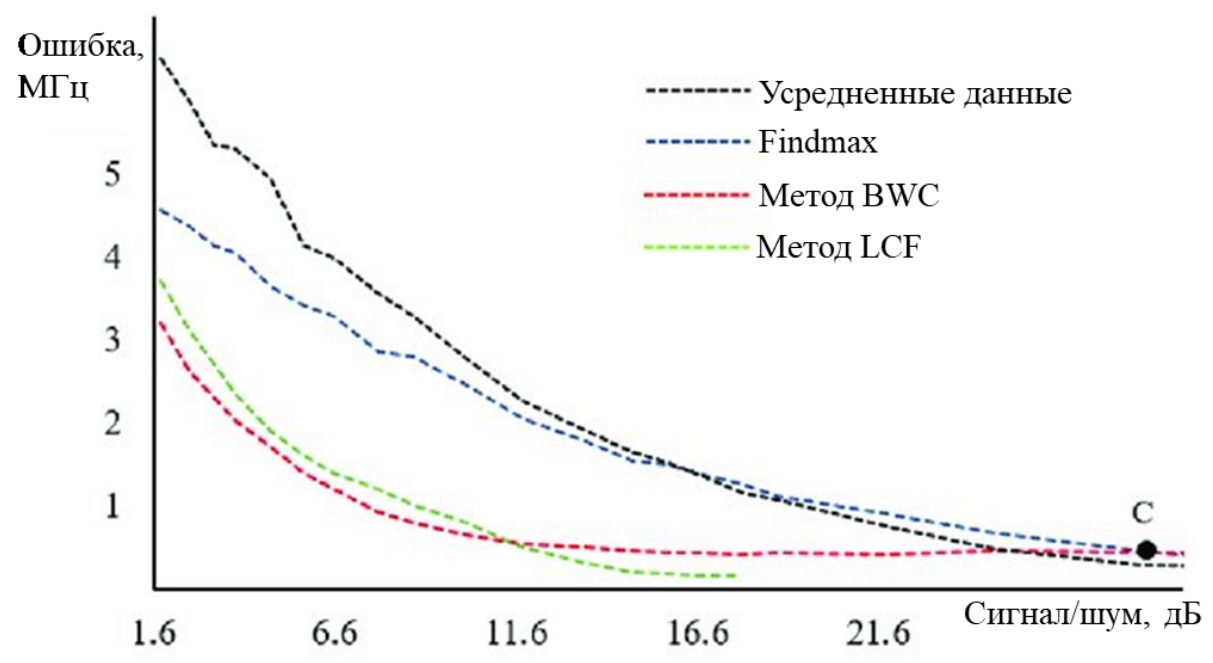

Рис. 8. Ошибка отклонения бриллюэновского сдвига на реальных измерениях при высоком отношении «сигнал-шум» 
Результаты классического расчета положения максимума BGS тремя методами, совместно со значением SNR спектра, а также шириной области артефакта в спектре подаются на вход нейронной сети с прямой связью (многослойный персептрон).

Нейронная сеть с прямой связью, используемая в работе, состоит из одного входного слоя, состоящего из пяти нейронов, двух скрытых слоев и выходного слоя, состоящего из одного нейрона (рис. 9).

Обучение сети производилось на сгенерированных спектрах по принципу обратного распространения ошибки. При этом способе обучения выходное значение сети сравнивается с правильным значением, а ошибка, представляющая собой разницу этих значений, возвращается через сеть. При этом корректируются значения весов связей между нейронами для последующего уменьшения ошибки.

В результате работы сети было получено улучшение определения максимума бриллюэновского спектра около $10 \%$. Сравнительные характеристики методов расчета максимума для смоделированных спектров приведены в табл. 2.

\begin{tabular}{|c|c|}
\multicolumn{1}{c}{ Таблица 2} \\
\begin{tabular}{|c|c|}
\multicolumn{2}{c}{ Точность методов определения } \\
максимума спектра
\end{tabular} \\
\hline Метод & $\begin{array}{c}\text { Стандартное отклонение } \\
\text { ошибки }\end{array}$ \\
\hline BWC & 0,0072 \\
\hline CCM & 0,0071 \\
\hline LCF & 0,0086 \\
\hline $\begin{array}{c}\text { Комбинированный } \\
\text { (Нейросеть) }\end{array}$ & 0,0064 \\
\hline
\end{tabular}

Описанные ранее элементы и подэлементы системы находят свое место в следующей АСИ для применения в лабораторных и производственных приложениях (рис. 10):

Здесь в начале процесса производится анализ поляризационных дефектов, которые могут иметь место после процесса намотки оптического волокна. На вход модели подаются распределение показателя преломления оптического волокна, полученное при помощи распределенного анализа, а также угол закрутки оптического волокна. Предполагаемая величина закрутки оптического волокна должна быть количественно определена для каждой производственной линии отдельно.

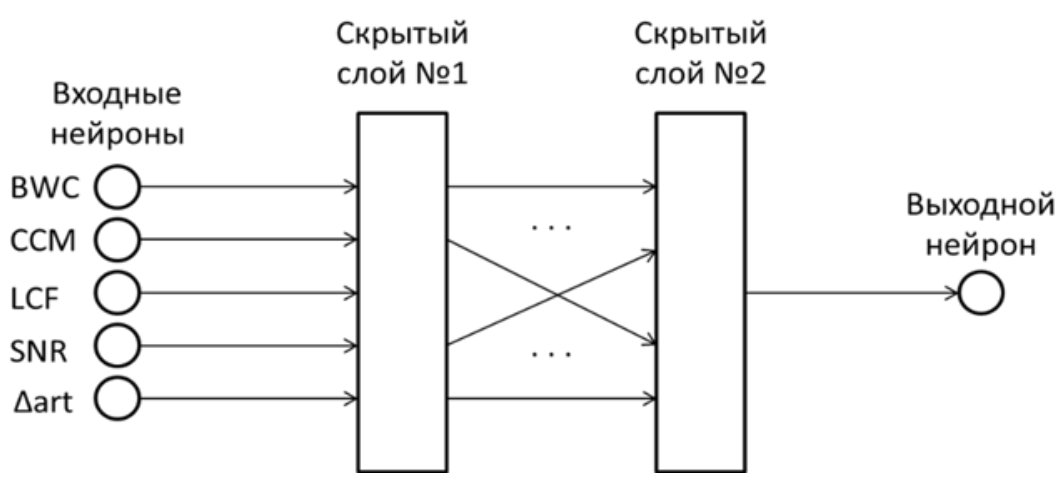

Рис. 9. Структура нейронной сети

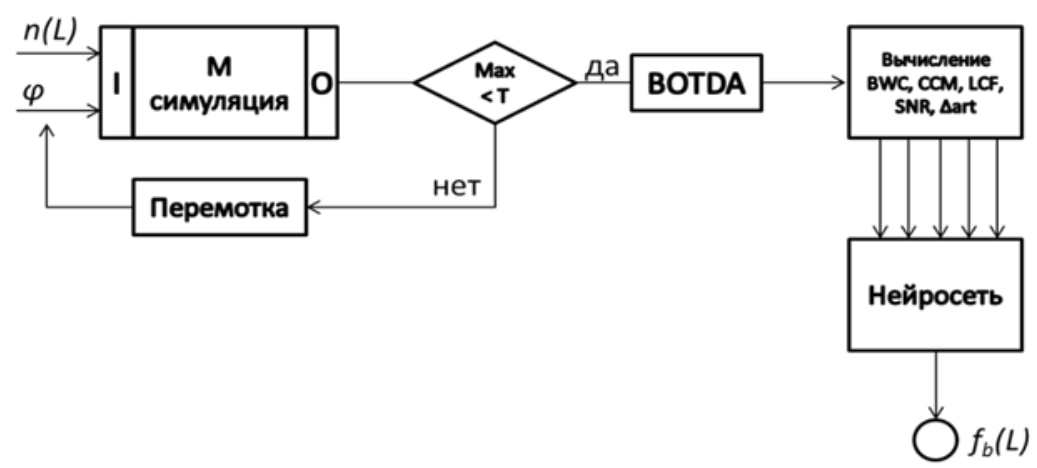

Рис. 10. Структура автоматизированной системы обработки бриллюэновских спектров 
Максимальная поляризационная погрешность не должна превышать некий порог, который будет выбран в соответствии с диапазоном приложений датчика.

Затем производится измерение при помощи бриллюэновского анализатора, в результате чего будет получен набор спектров вдоль волоконной линии. Эти спектры подвергаются анализу при помощи трех методов оценки положения максимума спектра. На последнем этапе полученные данные подаются на вход нейронной сети, в результате чего на выходе системы получается искомое значение положения максимума бриллюэновского спектра.

\section{Заключение}

В работе рассмотрены и проанализированы элементы и концепция автоматизированной системы контроля качества специальных волоконных световодов, сохраняющих состояние поляризации излучения, основанные на современных подходах к получению и обработке информации, получаемой при помощи бриллюэновской рефлектометрии.

Представлены имитационные модели и принцип работы методов обработки информации с бриллюэновских датчиков. Предложена программно-аппаратная система, включающая модули сбора информации на ранних этапах производства и комбинированный нейросетевой модуль, позволяющие с повышенной точностью определять оптические параметры изделия для более раннего принятия решения о соответствии изделия требуемым параметрам.

Применение элементов АСИ позволило увеличить точность определения максимума сдвига бриллюэноской частоты на величину порядка $10 \%$.

\section{Библиографический список}

1. Константинов Ю.А., Крюков И.И., Поскребышев М.М., Харламова Н.А. Автоматизированный сбор данных при исследовании характеристик волоконных световодов на этапах производства // Научно-технические ведомости СПбГПУ. Информатика. Телекоммуникации. Управление. - 2011. - № 6-2(138). - С. 30-34.

2. Константинов Ю.А., Крюков И.И., Поскребышев М.М., Харламова Н.А. Телевизионная система измерения размеров заготовки волоконных световодов в ходе процесса химического парофазного осаждения // Научно-технические ведомости СПбГПУ. Информатика. Телекоммуникации. Управление. - 2010. - № 6 (113). - С. 155-158.

3. Константинов Ю.А., Первадчук В.П. Автоматизация производства специальных волоконных световодов с применением эмуляторов физических процессов обратного рассеяния // Автоматизация и современные технологии. - 2013. - № 8. - С. 3-9.

4. Jasenek J., Cermak O. Optical reflectometry with synthesized coherence function// Proceedings Volume 4016, Photonics, Devices, and Systems; (1999) / Photonics Prague '99 - 1999.

5. Soller B., Gifford D., Wolfe M., Froggatt M. High resolution optical frequency domain reflectometry for characterization of components and assemblie // Opt Express. - 2005. - Vol. 13 - № 2. - P. 666-674.

6. Soller B.J., Wolfe M., Froggatt M.E. Polarization resolved measurement of Rayleigh backscatter in fiber-optic components// OFC Technical Digest - 2005

7. Madsen K., Nielsen H.B., Tingleff O. Methods for non-linear least squares problems// Informatics and Mathematical Modeling Technical University of Denmark, 2nd ed. London, U.K.: Inf. Math. Model., 2004. - 58 p.

8. Seber G., Wild C.J. «Nonlinear Regression» // New York, - NY, USA: Wiley - 2003.

9. Jones R.C. New calculus for the treatment of optical systems // Journal of the Optical Society of America - 1941. - Vol. 31. - P. 488.

10. Krivosheev A.I., Konstantinov Yu.A., Barkov F.L., Pervadchuk V.P. Comparative Analysis of the Brillouin Frequency Shift Determining Accuracy in Extremely Noised Spectra by Various Correlation Methods // Instruments and Experimental Techniques - 2021. - Vol. 64. - № 5. - P. 715-719.

11. Barkov F.L.; Konstantinov Y.A.; Krivosheev A.I. A Novel Method of Spectra Processing for Brillouin Optical Time Domain Reflectometry // Fibers - 2020. - Vol. 8. - P. 60.

12. Кривошеев А., Барков Ф., Константинов Ю. КОНТРОЛЬ ВОЛС методом бриллюэновской рефлектометрии: проблемы и возможные решения // Первая миля. 2020. - № 7-8 (92). - C. 38-45.

13. Barkov F.L., Konstantinov Yu.A., Burdin V.V., Krivosheev A.I. Theoretical and Experimental Estimation of the Accuracy in Simultaneous Distributed Measurements of Temperatures and Strains in 
Anisotropic Optical Fibers Using Polarization-Brillouin Reflectometry // Instruments and Experimental Techniques. - 2020. - Vol. 63. - № 4. - P. 487-493.

14. Belokrylov M.E., Konstantinov Yu.A., Latkin K.P., Claude D., Seleznev D.A., Stepin A.A., Konin Yu.A., Shcherbakova V.A., Kashina R.R. An all-fiber time domain reflectometer for measuring the length of active erbium doped optical fibers // Instruments and Experimental Techniques. - 2020 Vol. 63. - № 4. - P. 481-486.

\title{
CREATING AN AUTOMATED SYSTEM ELEMENTS FOR MEASURING THE OPTICAL FIBERS BRILLOUIN SHIFT IN INDUSTRIAL PRODUCTION
}

\author{
A.I. Krivosheev, Yu.A. Konstantinov \\ Perm Federal Research Center UB RAS
}

\section{For citation:}

Krivosheev A.I., Konstantinov Yu.A. Creating an automated system elements for measuring the optical fibers brillouin shift in industrial production // Perm Federal Research Center Journal. - 2021 - № 4 - P. 37-48. https://doi.org/10.7242/2658$705 X / 2021.4 .4$

The paper presents a modern consideration of developing an automated quality control system for special optical fibers that maintain the state of radiation polarization. A simulation model of reflectometry is presented taking into account the longitudinal twisting of optical fiber. The principle of system operation is described considering that the use of a new method for Mandelstam Brillouin scattering spectra in conjunction with already known methods, combined in a neural network to increase the accuracy of the system.

Keywords: automated measurement system (AMS), optical reflectometry, optical fibers, automated control, Brillouin reflectometry, polarization.

\section{Сведения об авторах}

Кривошеев Антон Иванович, младший научный сотрудник, Пермский федеральный исследовательский центр УрО РАН (ПФИЦ УрО РАН), 614990, г. Пермь, ул. Ленина 13а; e-mail: antokri@ya.ru

Константинов Юрий Александрович, кандидат технических наук, заведующий лабораторией фотоники, Пермский федеральный исследовательский центр УрО РАН (ПФИЦ УрО РАН), 614990, г. Пермь, ул. Ленина 13a; e-mail: yuri.al.konstantinov@ro.ru 